\title{
36 REVIEW OF CT COLONOGRAPHY: REAL-LIFE EXPERIENCE OF ONE THOUSAND CASES IN A TERTIARY REFERRAL CENTRE
}

A E Smyth, C F Healy, P MacMathuna, H M Fenlon Departments of Gastroentertolgoy and Radiology, Mater Misericordiae University Hospital, Dublin, Ireland

10.1136/gutjnl-2013-305143.36

Introduction CT Colonography is increasingly being utilised to investigate patients with lower gastrointestinal symptoms and in screening for colorectal carcinoma in the at risk population.

Aims/Background This review aimed to quantify results from a real life experience of 1000 CT Colonography examinations in a tertiary referral hospital campus.

Method Data on patient demographics, indications and findings were analysed retrospectively from a dedicated database.

Results Over a five year period (January 2008-December 2012) 1000 CT Colonography examinations were performed. The median age was 70 years (IQR 60-79). Patients were symptomatic in $86 \%$ of cases, screening accounted for $7 \%$ and surveillance in patients with a history of polyps or colorectal cancer for $7 \%$. In $45 \%$ of patients it was documented that the study was performed following an incomplete optical colonoscopy.

CT Colonography had normal or benign colonic findings in $75 \%$ of patients. $6 \%$ had incomplete examinations performed. Neoplasia was observed in 14\%: colorectal carcinoma $6 \%$, polyps $>1 \mathrm{~cm}$ in $3 \%$ and $5 \%$ were found to have $<3$ polyps of 6-9 mm. 5\% had findings of wall thickening or strictures that were suspected to be benign but required further endoscopy and biopsy.

There was an extracolonic finding that required further evaluation in $19 \%$ with $6 \%$ found to have a potentially clinically significant finding.

There were no significant procedure related complications. Conclusion This review describes the real life experience of CT Colonography in a largely elderly, symptomatic patient group. In this patient group, CT Colonography safely confirmed the need for no further bowel tests in $75 \%$, identified colorectal cancer in $6 \%$ and clinically significant extracolonic findings in $6 \%$ of cases without any significant complications. 